\title{
MLAMAN: A NOVEL MULTI-LEVEL AUTHENTICATION MODEL AND PROTOCOL FOR PREVENTING WORMHOLE ATTACK IN MOBILE AD HOC NETWORK
}

\author{
Tu T. Vo ${ }^{1}$, Ngoc T. Luong ${ }^{1,2}$, Doan Hoang ${ }^{3}$ \\ ${ }^{1}$ Faculty of Information Technology, Hue University of Sciences, Hue University, Viet Nam \\ ${ }^{2}$ Faculty of Mathematics and Informatics Teacher Education, Dong Thap University, Viet Nam \\ ${ }^{3}$ Faculty of Engineering and Information Technology, the University of Technology Sydney, Australia \\ ${ }^{1}$ vttu@hueuni.edu.vn; ${ }^{2}$ ltngoc@dthu.edu.vn; ${ }^{3}$ Doan.Hoang@uts.edu.au
}

\begin{abstract}
Wormhole attack is a serious security issue in Mobile Ad hoc Network (MANET) where malicious nodes may distort the network topology and obtain valuable information. Many solutions, based on round trip time, packet traversal time, or hop-count, have been proposed to detect wormholes. However, these solutions were only partially successful in dealing with node highspeed mobility, variable tunnel lengths, and fake information by malicious nodes. To address those issues, this paper proposes a novel multi-level authentication (MLA) model and protocol (MLAMAN) for detecting and preventing wormhole attacks reliably. MLAMAN allows all intermediate nodes to authenticate control packets on a hop-by-hop basis and at three levels: 1) the packet level where the integrity of the packets can be verified, 2) the node membership level where a public key holdermember can be certified, and 3) the neighborhood level where the neighborhood relationship between nodes can be determined. The novelty of the model is that it prevents malicious nodes from joining the network under false information and pretense. It detects wormhole nodes effectively under various scenarios including variable tunnel lengths and speeds of moving nodes. The effectiveness of our approach is confirmed by simulation results through various scenarios.
\end{abstract}

Keywords. AODV; MANET; MLA; MLAMAN; mobile ad hoc network; network security

\section{Introduction}

A Mobile Ad hoc Network (MANET [5]) is a collection of wireless mobile nodes without network infrastructures, routers or access points. The topology of the network can change unpredictably and frequently because of nodes joining or leaving. In a MANET, nodes communicate with neighbors to discover and maintain routes to their destinations. Data transfer from a source node to a destination node can be routed through intermediate nodes, which act both as hosts or routers. A network routing protocol in a MANET specifies how nodes in the network communicate with one another. It enables a node to discover and maintain the routes as needed between itself and other nodes. Many routing protocols have been developed for MANETs [3]; among them, Ad hoc On-demand Distance Vector (AODV) [21] and Dynamic Source Routing (DSR) [10] are the most important protocols. Because of the ad hoc nature, Denial of service (DoS) is a serious issue in MANET. DoS attacks aim to deny a user of a service or a resource he/she would normally expect to receive. Disrupting the routing services at the network layer is an example of DoS [22] where a malicious node 
tries to deplete resources of other nodes. Other types of DoS include Blackhole [24], Sinkhole [19], Grayhole [4], Flooding [25], Whirlwind [20], and Wormhole [12].

The wormhole attacks in Ad hoc Networks are described in [9][12][14] cite where the authors categorize several types of packet tunneling wormhole attacks, including wormhole through the tunnel (called out-of-band channel - OB), wormhole using encapsulation, wormhole using packet relay, and wormhole with high power transmission. Such wormhole attacks may operate in two modes: Hidden Mode (HM) and Participation Mode (PM). In HM, malicious nodes are hidden from normal nodes, which on receiving a packet they simply forward the packet without processing it. By doing so, the malicious nodes are invisible as they never appear in the routing tables of their neighbors. In contrast, in PM, malicious nodes are visible during the route discover process because they process control packets just like other normal nodes. These malicious nodes appear in the routing tables of their neighbors and the hop-count $(\mathrm{HC})$ values increase when control packets are routed. This type of attacks can easily be carried out with on-demand routing protocols, typically the AODV routing protocol to eavesdrop information.

Previous researches on wormhole attacks mainly focus on detection algorithms that rely on Geographical Location, Round Trip Time, Packet Traversal Time, Hop-Count information, or Digital signatures. These algorithms, however, have many weaknesses: With geographical location based approaches such as LBK [15] or SeRLoc [16], nodes continuously broadcast location data (GPS information) and all transmissions between node pairs are encrypted. On the other hand, the TIK [6] solution rely on synchronization among all nodes. These solutions thus incur high communication overhead. With time analysis based approaches such as DelPHI [1], MHA [9], and TTHCA [12][13], a malicious node may collaborate with the route discovery process but deliberately provides fake information in the control packets. With digital signature based approaches such as SAODV [18] which does not have a key management mechanism, malicious nodes can pass over the security by using fake keys. On the other hand, ARAN [23] supports key management, but it is not able to detect wormhole node under HM mode (according to [8]). This paper proposed MLAMAN, a new MANET wormhole detection model that comprises a multi-level authentication (MLA) mechanism, an MLA-secured routing protocol, and a node membership certification protocol. The MLA mechanism uses digital signatures with the RSA [2] public key encryption and $\mathrm{SHA}_{1}$ [11] hashing function. With MLA, all intermediate nodes can authenticate control packets on a hop-by-hop basis and at three levels: 1) the packet level where the integrity of the packets can be verified, 2) the node membership level where a public key holder-member can be certified, and 3) the neighborhood level where the neighborhood relationship between nodes can be determined. The MLAMAN protocol is a modified version of the AODV to incorporate the MLA mechanism. The node membership certification protocol allows nodes to participate in the routing procedure. It is demonstrated that the proposed solution is effective in wormhole detection under various network scenarios, and prevents malicious node from taking part in the route discovery process with fake information.

The remainder of this article is structured as follows. Section 2 discusses related work. Section 3 describes the proposed MLAMAN model for wormhole detection with multi-level authentication (MLA) mechanism. The proposed MLAMAN protocol (MLA mechanisms for Mobile Ad hoc Network) by integrating the MLA into the route discovery procedure of AODV protocol. A public key management method and protocol to provide Membership 
Certification (MC) for a node before it can participate in the route discovery process. Section 4 presents the performance evaluation results by simulation using NS2 [7]. Finally, section 5 concludes the paper and provides a discussion on future work.

\section{Related works}

This section summarizes related work that focuses on location-based, time-based, and digital signature-based approaches in detecting and preventing wormhole attacks in MANETs. On geographical location based approach. The authors in [15] described a graph theoretic model to characterize the wormhole attack and prevent wormholes. They used a Local Broadcast Key (LBK) method to install a secure Ad-hoc Network against wormhole attacks. Two types of nodes are involved: guard nodes and regular nodes. The Guard nodes continuously broadcast location data obtained from localization systems such as the Global Positioning System (GPS) or SeRLoc [16]. Regular nodes calculate their location relative to the guards' beacons and by doing so they can detect abnormal resend transmission by the wormhole attackers. All transmissions between node pairs are encrypted by the local broadcast key of the sending end and decrypted at the receiving end. This approach is suitable for networks with fixed and static topology wireless sensor networks. For dynamic topologies, the solution is not effective as its performance suffers badly due to large time delay variations and communication overhead caused by continuous broadcast of location data.

On time analysis based approach. The authors in [6] described the TIK protocol for detecting wormhole attacks. TIK uses Packet Leashes solution that appends timing information to a packet to limit its admissible transmission distance. Thus, a wormhole attack is detected when packets are delivered at a much shorter time than possible through expected valid routes. TIK depends on a strict synchronization among all nodes. As synchronization is difficult and resource-consuming, such detection method become less effectiveness in highspeed mobile networks. The authors in [1] described the Delay Per Hop Indication (DelPHI) solution for detecting wormhole attacks. The idea is to allow the source node to receive the route reply packets on many routes and calculates the round trip time (RTT) per route. It is assumed that a route with a small number of hops has a small RTT, so the route that has a higher RTT per hop count than a pre-calculated threshold is considered a wormhole route. However, in dynamic environments where the network loads are unpredictable and nodes move rapidly, the RTTs are highly variable, the proposed solution becomes less reliable. The authors in [9] proposed the multi-hop count analysis (MHA) solution based on hop counts. MHA does not require Round Trip Time (RTT) measurement. MHA modifies the AODV route discovery protocol to identify several unique routes between the source and the destination nodes. A route with a much lower $\mathrm{HC}$ value than other routes is then assumed to include a wormhole and is avoided. The authors in [12] presented a wormhole detection algorithm based on Traversal Time and Hop Count Analysis (TTHCA) for the AODV routing protocol. TTHCA obtains the packet transversal time (PTT) by subtracting the highly variable processing times of nodes along the route from the RTT and provides a more reliable wormhole detection performance with low error rates, and small computational costs. However, the TTHCA detection ability to malicious nodes is restricted because the PTT of packet is seriously affected by nodes moving at fast speeds. An improved TTHCA to identify time measurement tampering in traversal time and hop count analysis wormhole 
detection algorithm, is described in [13].

On digital signature based approach. Many proposed solutions deployed cryptographic techniques to protect routing packets and detect wormholes. The Secure AODV (SAODV) [18] is proposed to prevent malicious nodes from fabricating the HC number and the Sequence Number (SN) in route discovery packets. However, SAODV only supports end-to-end certification and not hop-by-hop, as a consequence, an intermediate node cannot certify packet from its preceding node. In addition, because SAODV does not have a key management mechanism for node, so malicious nodes can pass over security by using fake keys. The authors in [23] proposed the Authenticated Routing for Ad hoc Networks (ARAN) protocol for detecting and preventing attacks on insecure protocols. Different from SAODV, route discovery packet (RDP) in ARAN is signed and certified at all hop-by-hop nodes. ARAN supplements the public key management mechanism to provide authentication and non-repudiation services. However, it is not able to detect wormhole node under HM mode. Furthermore, ARAN assumes that there is no way to way to guarantee that one path is shorter than another in terms of hop count. Accordingly, ARAN does not guarantee a shortest path, but offers a quickest path which is chosen by the RDP that reaches the destination first. That means that ARAN is unable to identify the routing costs through intermediate relaying nodes.

\section{MLAMAN - A Multi-Level Authentication Model for MANETs}

This section describes our proposed Multi-Level Authentication model for mobile ad hoc networks, MLAMAN consists of three components: a multi-level authentication mechanism for detecting and preventing both PM and HM wormhole attacks, a modified AODV protocol (MLAMAN routing protocol) for route discovery and route maintenance, and an auxiliary node membership certification protocol. The following subsections describe the three elements of the MLA model.

\subsection{The MLA mechanism}

The MLA mechanism is designed to authenticate routing packets (RREQ or RREP) on a hop-by-hop basis and at three levels: (1) Packet integrity level; (2) Node member certification level; (3) and Neighborhood verification level. Table 1 defines symbols used in the paper.

Table 1. Description of symbols

\begin{tabular}{|l|l|}
\hline Variable & Descriptions \\
\hline \hline $\mathrm{MC}_{N_{\delta}}$ & Membership certificate of node $N_{\delta}$ \\
\hline $\mathrm{T}_{M C}$ & Time interval for $\mathrm{N}_{\text {center }}$ checks PKDB to provide the MC \\
\hline $\mathrm{N}_{\delta}$ & Node labeled $N_{\delta}$ \\
\hline$k_{N_{\delta}}+, k_{N_{\delta}}-$ & Public and private keys of node $N_{\delta}$ \\
\hline $\mathrm{En}(\mathrm{v}, \mathrm{k})$ & Encryption of value $\mathrm{v}$ using key $\mathrm{k}$ \\
\hline $\mathrm{De}(\mathrm{v}, \mathrm{k})$ & Decryption of value $\mathrm{v}$ using key $\mathrm{k}$ \\
\hline $\mathrm{H}(\mathrm{v})$ & Hash value of $\mathrm{v}$ \\
\hline $\mathrm{IP}$ & Address of node $\delta$ \\
\hline PKDB & Public key database in node $\mathrm{N}_{\text {center }}$ \\
\hline
\end{tabular}




\subsubsection{Packet integrity verification}

The integrity verification process is designed to prevent a malicious node to tamper with the control packet when it is relayed from hop to hop. Under the PM mode, malicious nodes may deliberately alter the contents of the routing packets. The main idea is for a node $\left(N_{i}\right)$ to verify the integrity of the RREQ packet (or RREP) it receives from a source node or an intermediate node $N_{j}$. The packet verification value is obtained as follows. First, the sending node $N_{j}$ uses $H$ to hash all protected fields of the RREQ (or RREP) packet and then encrypts the resulting hash value using its private key as shown in eqn 1 . The encrypted value is saved into the $\mathrm{VC}$ field of the packet (RREQ or REP) before broadcasting it to its neighbor $N_{i}$.

$$
P . V C=\operatorname{En}\left(H(\text { P.Fields }\{V C\}), k_{N_{j}}-\right)
$$

Where $P$ is RREQ or RREP packets.

Algorithm 1 shows steps that $N_{i}$ verifies the integrity of the packet RREQ (or RREP) on receiving from a source (or an intermediate) node $N_{j}$. Node $N_{i}$ uses the public key $k_{N_{j}}+$ to decrypt the VC field value of packet (P.VC). Protected fields of the packet are hashed by $N_{i}$ using the $H$ function. If the hask value val2 matches val1 the integrity of the packet is verified, otherwise else the packet has been modified, and it can be concluded that the preceding node (node $N_{j}$ ) is a PM wormhole node.

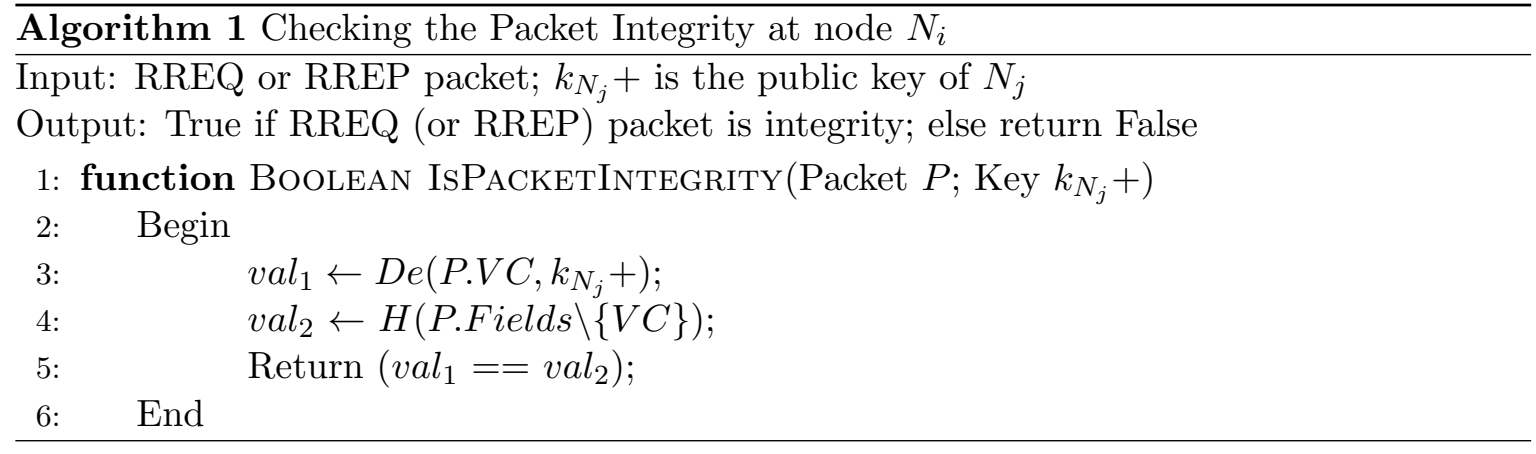

\subsubsection{Node member authentication}

As with other solutions that deployed cryptographic techniques, each node in the environment has a private key and a public key. The proposed solution also assumes that for a node to participate in the route discovery process it has to be certified and its certificate can be verified by any other node with the proposed procedure. This node member authentication excludes malicious nodes from the routing process and overcomes the weakness from SAODV. We use a reliable node named $\mathrm{N}_{c e n t e r}$ to manage and provide the Membership Certification (MC) to all nodes using the MCP and $M C_{A C K}$ packets. Section 3.3. will discuss the procedure and the auxiliary protocol for this node membership certification.

Definition 1: Membership Certification is provided for all nodes automatically by the $\mathrm{N}_{\text {center }}$ and it is added to the RREQ or RREP packet while node participates in the discovery route process. MC of node $N_{\delta}$ is calculated by first encrypting the hash value of the member's node address $\left(I P_{N_{\delta}}\right)$ and public key $\left(k_{N_{\delta}}+\right)$ with the private key of the $\mathrm{N}_{c e n t e r}$ and then 
encrypting the encrypted result of the first step with the private key of the member node $N_{\delta}$ as shown in eqn 2 .

$$
M C_{N_{\delta}}=\operatorname{En}\left(\operatorname{En}\left(H\left(I P_{N_{\delta}}, k_{N_{\delta}}+\right), k_{N_{c e n t e r}}-\right), k_{N_{\delta}}-\right)
$$

Algorithm 2 shows steps to verify MC when node $N_{i}$ receives a RREQ (or RREP) packet sent (or forwarded) by node $N_{\delta}$. Node $N_{i}$ decrypts the MC field of the packet (P.MC) using the public key of node $N_{\delta}$ and then decrypts the resultant with the public key of node $N_{\text {center }}$. If the value after decryption matches the hash value of $N_{\delta}$ node address then $N_{\delta}$ is a valid member node. Otherwise, the packet is dropped because the membership of sending node $N_{\delta}$ cannot be verified (either the node is unknown or has not been MCed by Ncenter). Note that the member nodes authentication process is performed only when the integrity of the packet RREQ (or RREP) has been verified.

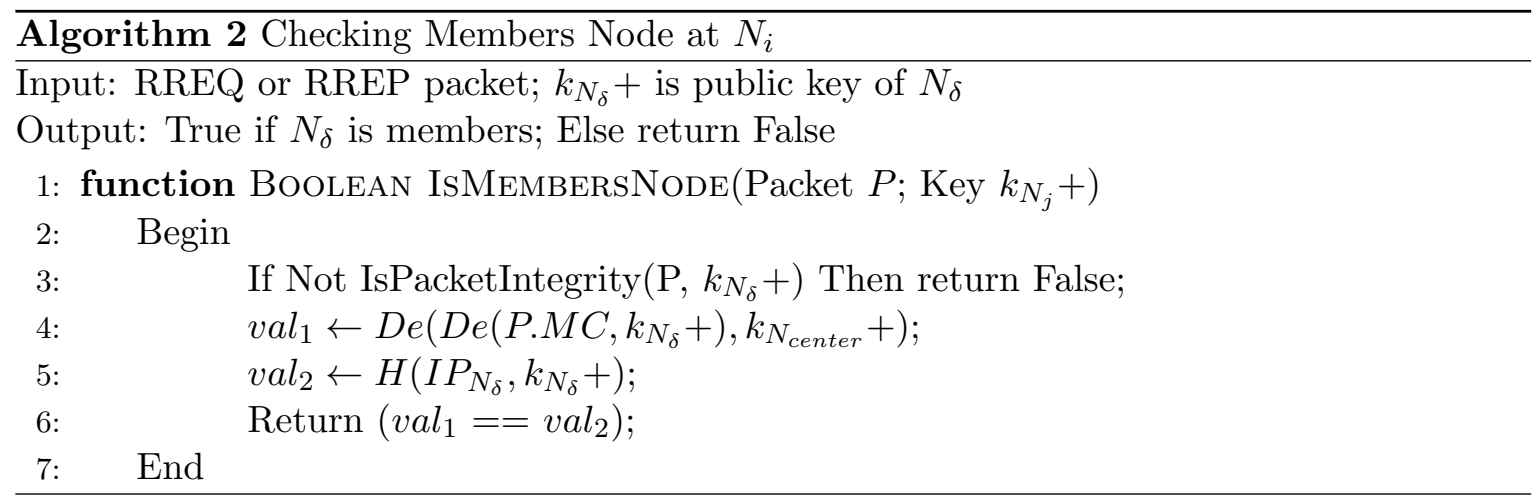

\subsubsection{Neighborhood relationship verification}

The proposed neighborhood relationship determination allows a member node to detect a route with wormhole node. When $N_{i}$ receives RREQ (or RREP) packet from $N_{j}$, if both of $N_{i}$ and $N_{j}$ nodes are not physically neighbors, a HM wormhole node must have operated on the discovered route.

Definition 2: Two nodes $\left(N_{i}\right.$ and $\left.N_{j}\right)$ are geographic and physical neighbors of each other if they are within their transmission radius. Explicitly, $N_{i}$ and $N_{j}$ are neighbors only if $d\left(N_{i}, N_{j}\right) \leqslant \min \left(R_{N_{i}}, R_{N_{j}}\right)$, where $R_{N_{\delta}}$ is maximum transmission radius of $N_{\delta}$ and $d\left(N_{i}, N_{j}\right)$ is Euclidean distance between $N_{i}$ and $N_{j}$ as given by eqn 3 , and $\left(x_{N_{\delta}}, y_{N_{\delta}}\right)$ is the location coordinate of node $N_{\delta}$.

$$
d\left(N_{i}, N_{j}\right)=\sqrt{\left(x_{N_{i}}-x_{N_{j}}\right)^{2}+\left(y_{N_{i}}-y_{N_{j}}\right)^{2}}
$$

Example 1: In the network topology shown in Figure 1a), both of nodes $N_{1}$ and $N_{2}$ are neighbors in the physical sense because the distance between $N_{1}$ and $N_{2}$ is less than (or equal to) the minimum transmission radius of the two nodes. In MANETs, the location of a node changes due to its mobility nature. We adopt the use of GPS to obtain node location as described in [14][15]. 


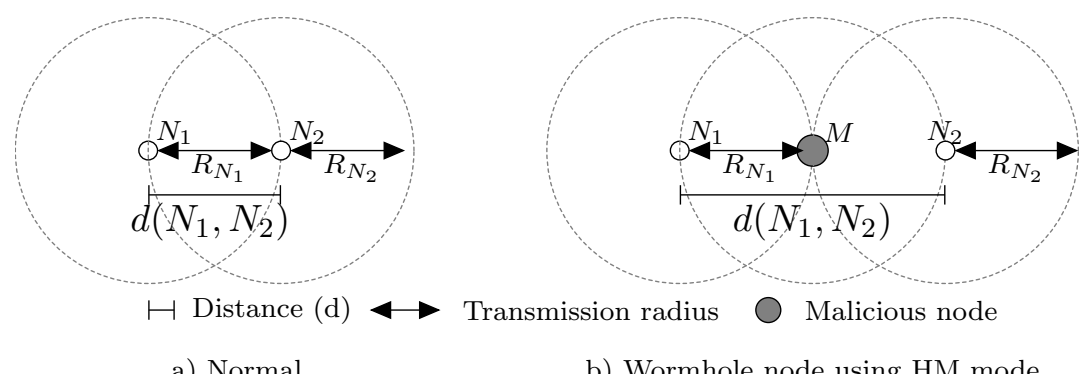

Figure 1. Physical neighbor nodes

Example 2: In wormhole HM mode attack, malicious nodes are hidden from normal nodes. As shown in Figure 1b), a wormhole node M, on receiving the RREQ (or RREP) from $N_{1}$, it will forward the packet to $N_{2}$ without changing the packet. Hence, $N_{2}$ cannot detect the malicious node $\mathrm{M}$ with only packet integrity verification and member node authentication.

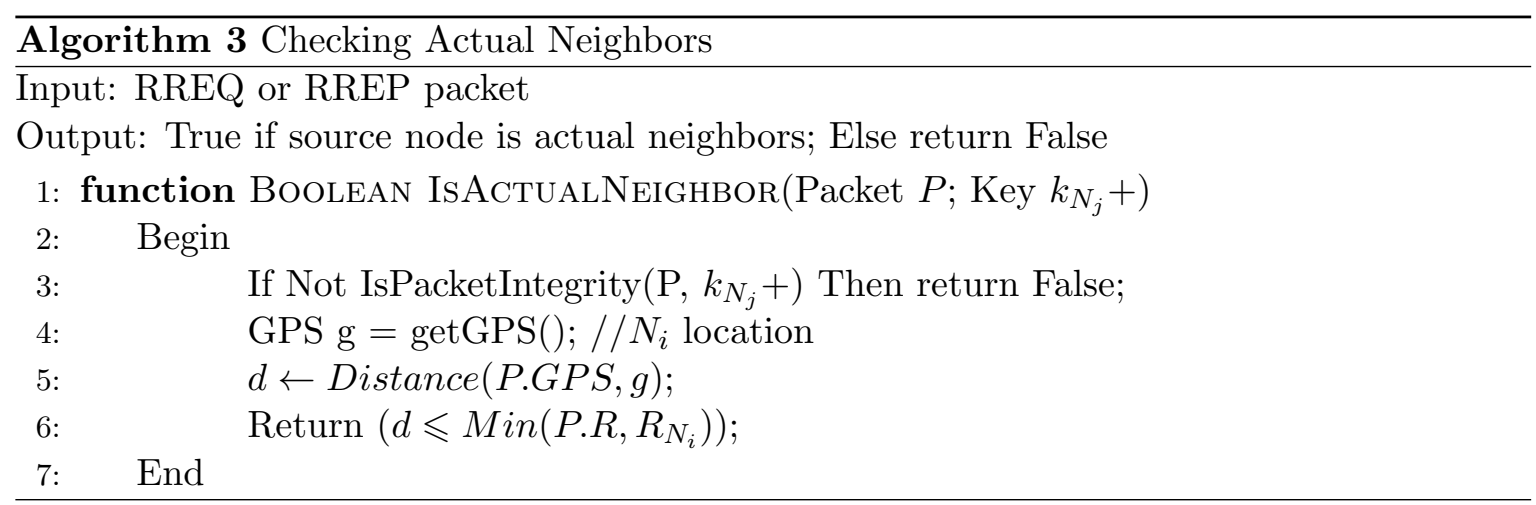

The procedure for determining if a node is a true physical neighbor of another node is shown in Algorithm 3. In order to calculate the distance between the two nodes, $N_{j}$ saves the location information and maximum radio range into GPS and $\mathrm{R}$ fields of the packet before sending (or forwarding) the packet. At node $N_{i}$, after receiving the packet from node $N_{j}$, it checks if the distance between itself and $N_{j}$ is less than (or equal) the minimum of the radio range (R) of the two nodes then $N_{i}$ and $N_{j}$ are verified true neighbors, otherwise, there is a wormhole node on discovered route invisibly relaying the packet.

\subsection{MLAMAN protocol - A secure AODV route discovery with Multi-Level Authentication}

As part of the MLAMAN model, we propose MLAMAN protocol, a secure and enhanced AODV with built-in MLA mechanism for detecting and preventing wormhole attacks. Similar to AODV, MLAMAN protocol includes two phases: a broadcasting route request phase and an unicasting route reply phase. The control route packet structures of the new protocol (SecRREQ and SecRREP) extend the control packet structures of AODV (RREQ and RREP) to include five new fields (5NF): GPS, R, MC, KEY and $V C$ as shown in Figures 2. 


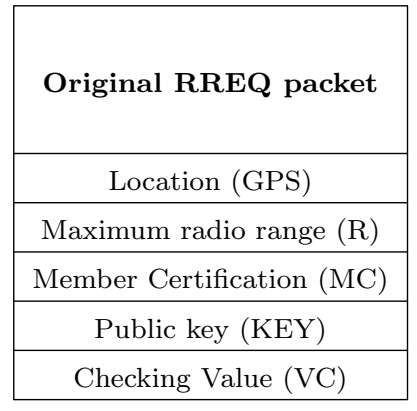

(a) SecRREQ

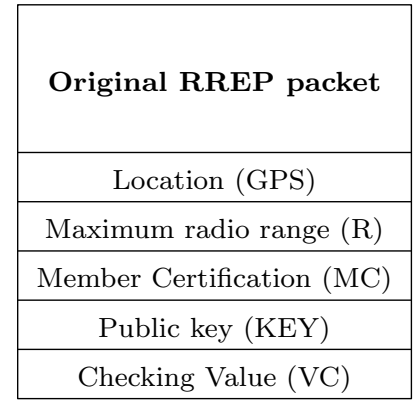

(b) SecRREP

Figure 2. The control route packet structures of MLAMAN protocol

\subsubsection{Broadcasting route request packet phase}

a) Generating SecRREQ packet: If the source node $\left(N_{S}\right)$ does not have a route to the destination node, it initiates a new route discovery process by broadcasting the SecRREQ packet to its neighbor nodes as in (4).

$$
N_{S} \text { broadcasts }: \text { SecRREQ } \leftarrow\left\{R R E Q^{*} \oplus 5 N F\right\}
$$

Where $R R E Q^{*}$ is the original RREQ packet of the AODV routing protocol and $5 \mathrm{NF}$ is the five new fields of the SecRREQ. These new fields contain the following information:

- SecRREQ.GPS $=N_{S}$ node GPS information;

- SecRREQ.R = $250 \mathrm{~m}$;

- SecRREQ.MC $=N_{S}$ node MC;

- SecRREQ.KEY = Source node public key $k_{N_{S}}+$;

- SecRREQ.VC = Encryption of H(SecRREQ.fields $\backslash\{\mathrm{VC}\})$ using $k_{N_{S}}-$;

b) Processing and forwarding SecRREQ packet: When a node receiving a SecRREQ packet, the node (say $N_{i}$ ) drops this packet if it has not been certified, otherwise, it tests the integrity of the packet, verifies the node membership of the sending node, and determines if the sending node is a true neighbor according to the MLA mechanism.

- If the integrity SecRREQ packet is not verified, $N_{i}$ drops the packet as the discovered route has been tampered by a malicious node under PM mode.

- If the SecRREQ packet is not sent by a certified member node, $N_{i}$ drops the SecRREQ packet;

- If the SecRREQ packet is not sent by a true neighbor node, $N_{i}$ drops the SecRREQ packet as the discovered route has been interfered by a malicious node under HM mode. 
If all the conditions are satisfied, and the current node is the destination, it generates and sends back the SecRREP packet; otherwise, it updates a reverse route toward the source node and the $5 \mathrm{NF}$ of the SecRREQ packet with the latest information before broadcasting the updated SecRREQ packet to its neighbors. Figure 3 describes the MLAMAN route request packet algorithm using MLA mechanism.

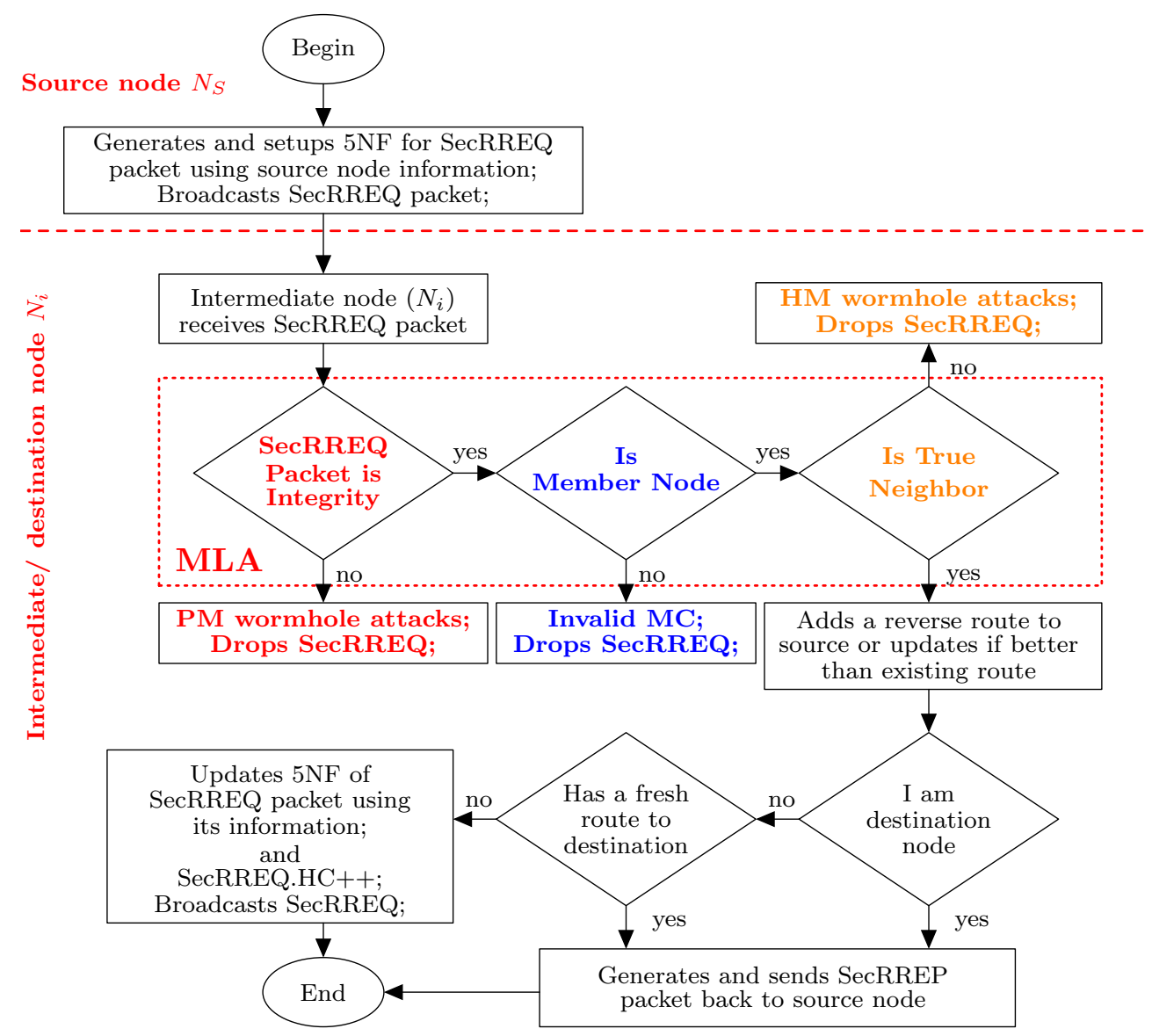

Figure 3. MLAMAN route request algorithm

\subsubsection{Unicasting route reply phase}

a) Generating SecRREP packet: Figure 4 describes route reply packet algorithm using the MLA mechanism. A node generates a SecRREP packet if it is either the destination $\left(N_{D}\right)$ or an intermediate $\left(N_{i}\right)$ which has a "fresh" route to the destination as in (5).

$$
N_{D}\left(\text { or } N_{i}\right) \text { unicasts }: \text { SecRREP } \leftarrow\left\{R R E P^{*} \oplus 5 N F\right\}
$$

Where $R R E P^{*}$ is the original RREP packet of the AODV routing protocol and $5 \mathrm{NF}$ is five new fields of the SecRREP. These new fields contain the following information: 
- SecRREP.GPS $=N_{D}$ (or $\left.N_{i}\right)$ node GPS information;

- SecRREP.R = $250 \mathrm{~m}$;

- SecRREP.MC $=N_{D}\left(\right.$ or $\left.N_{i}\right)$ node $\mathrm{MC}$;

- SecRREP.KEY = Public key $k_{N_{D}}+\left(\right.$ or $\left.k_{N_{i}}+\right)$;

- SecRREP.VC = Encryption of H(SecRREP.fields $\backslash\{\mathrm{VC}\})$ using $k_{N_{D}}-\left(\right.$ or $k_{N_{i}}-$ );

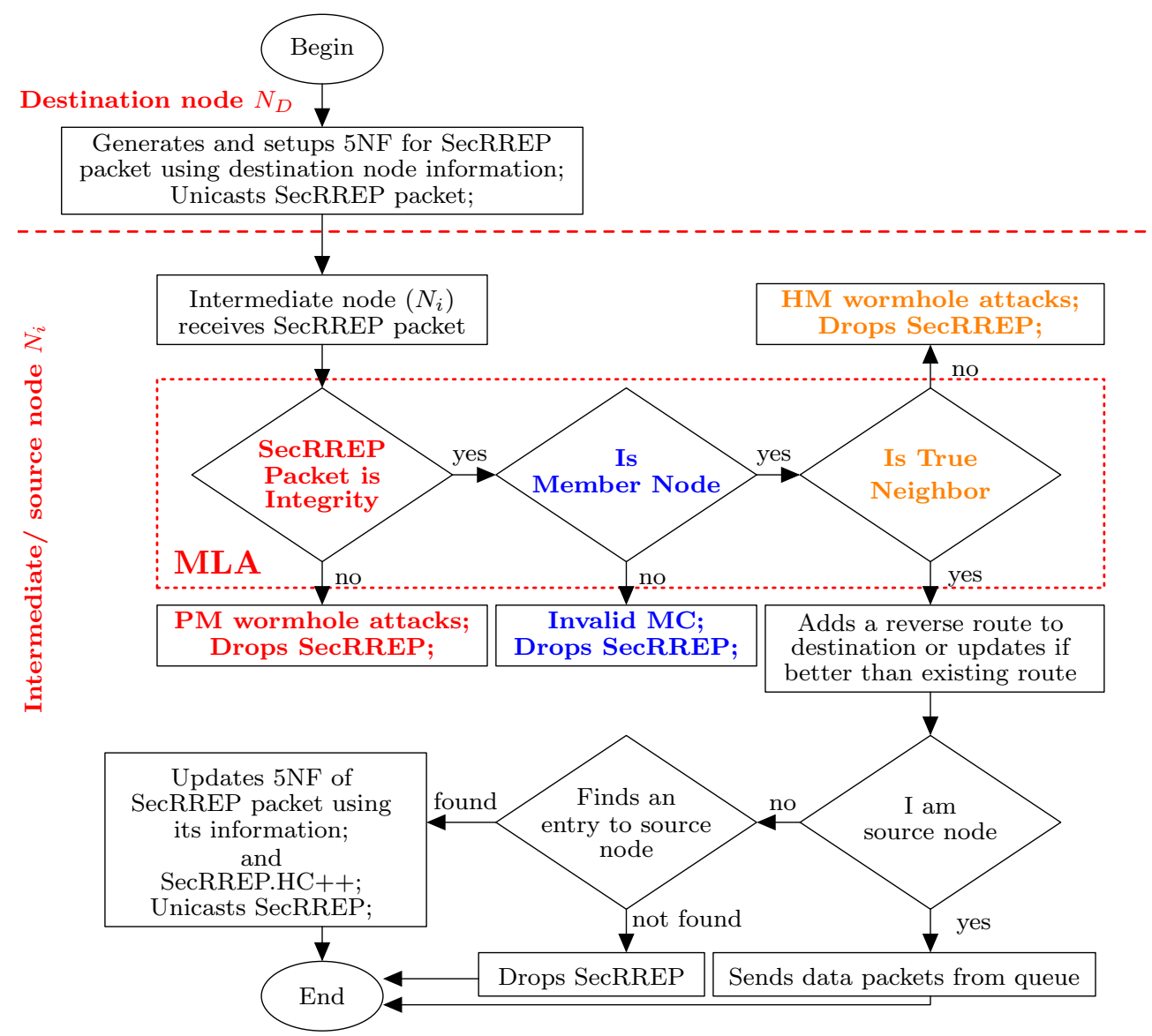

Figure 4. MLAMAN route reply algorithm

b) Processing and forwarding SecRREP packet: When a node receives a SecRREP packet, the node drops the packet if it has not been certified, otherwise, it tests the integrity of the packet, verifies the node membership of the sending node, and determines if the sending node is a true neighbor according to the MLA mechanism.

- If the integrity SecRREP packet is not verified, $N_{j}$ drops the packet as the discovered route has been tampered by a malicious node under PM mode. 
- If the SecRREP packet is not sent by a certified member node, $N_{j}$ drops the SecRREQ packet;

- If the SecRREP packet is not sent by a true neighbor node, $N_{j}$ drops the SecRREQ packet as the discovered route has been interfered by a malicious node under HM mode.

If all the conditions are satisfied, and the current node is the source node, it will send data packets to the destination node through the discovered route; otherwise, it updates a reverse route to the destination node and the $5 \mathrm{NF}$ of the SecRREP packet with the lastest information before sending the packet to the next hop the source node.

\subsection{MLAMAN auxiliary protocol and procedure for providing node member- ship certificate}

In a MLAMAN ad hoc network, any node can verify the certification of another node and only certified nodes can participate in the route discovery process. Figure 5 shows the procedure for providing the MC to member nodes. The MLAMAN administrator possesses a database of public keys (the PKDB) of all possible nodes that can join the ad hoc environment. Any node in the PKDB can be designated as the $\mathrm{N}_{c e n t e r}$ node. Some nodes in the PKDB may have already had their membership certified by the $\mathrm{N}_{c e n t e r}$ and some are yet to be certified. Periodically after $T_{M C}$ time interval, $\mathrm{N}_{\text {center }}$ checks the PKDB to see if all members have been provided with a membership certificate $(M C)$. If node $\left(\mathrm{N}_{\delta}\right)$ is not yet provided with an $\mathrm{MC}, \mathrm{N}_{\text {center }}$ broadcasts a membership certificate packet (MCP) to for the destination $\mathrm{N}_{\delta}$. On receiving the $\mathrm{MCP}$, node $\mathrm{N}_{\delta}$ sends an $\mathrm{MC}_{A C K}$ packet back to the $\mathrm{N}_{\text {center }}$ to confirm that it receives the MC. The procedure requires a PKDB and an auxiliary protocol for granting certificates to members of PKDB.

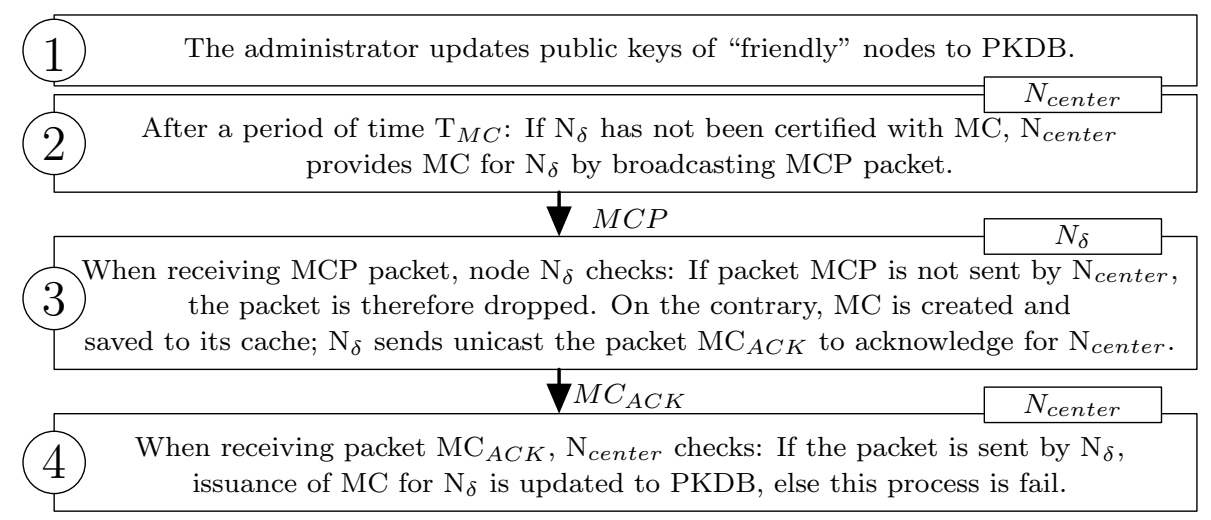

Figure 5. The process provides $\mathrm{MC}$ for member nodes

\subsubsection{The Public Key Database}

The administrator sets up a reliable node named $\mathrm{N}_{\text {center }}$ to provide $\mathrm{MC}$ for members. In $\mathrm{N}_{\text {center }}$, a public key database (PKDB) of all nodes is created with the structure shown 
in Table 2. Each record in the PKDB consists of: node address (Nodes), node public key $(\mathrm{Key}+)$, and node MC status. Where, two Nodes and Key+ attributes are updated directly by administrators to ensure that only legitimate nodes are provided with MC.

Table 2. Public key database structures

\begin{tabular}{|c|c|c|}
\hline Nodes & Key+ & Completed \\
\hline \hline$I P_{N_{1}}$ & $k_{N_{1}}+$ & yes \\
\hline$I P_{N_{2}}$ & $k_{N_{2}}+$ & yes \\
\hline$I P_{N_{3}}$ & $k_{N_{3}}+$ & no \\
\hline$\ldots$ & $\ldots$ & $\ldots$ \\
\hline$I P_{N_{n}}$ & $k_{N_{n}}+$ & yes \\
\hline
\end{tabular}

\subsubsection{MLAMAN membership certification protocol}

\section{a) Broadcasting MCP packet and saving MC}

$\mathrm{N}_{\text {center }}$ provides $\mathrm{MC}$ for a member node $\mathrm{N}_{\delta}$ by broadcasting a MCP packet. This protocol is again an enhanced protocol of AODV for broadcasting the RREQ packet. The structure of the MCP packet is similar to that of the RREQ packet and includes two new fields (2NF): $C E R$ and $V C$, as described in Figure 6(a).

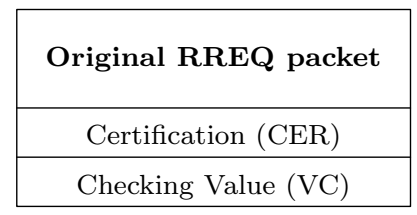

(a) MCP

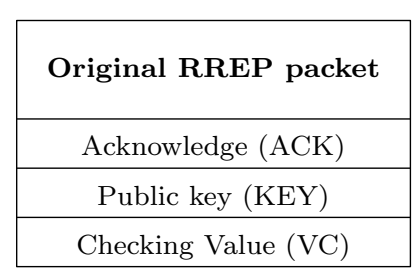

(b) $\mathrm{MC}_{A C K}$

Figure 6. The control packet structures of membership certification protocol

Generating MCP packet: Periodically after $\mathrm{T}_{M C}$ time interval, $\mathrm{N}_{\text {center }}$ checks to see if all node members are provided with an MC by broadcasting the MCP packet to all its neighbors as in (6).

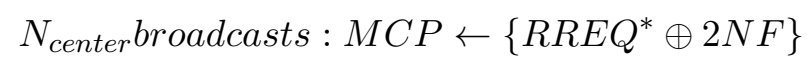

Where $R R E Q^{*}$ is the original RREQ packet of the AODV routing protocol and $2 \mathrm{NF}$ is two new fields of the MCP packet. The new fields contain the following information: the CER field as calculated by (7) and the packet integrity VC field.

$$
M C P . C E R=\operatorname{En}\left(\operatorname{En}\left(H\left(I P_{N_{\delta}}, k_{N_{\delta}}+\right), k_{N_{c e n t e r}-}\right), k_{N_{\delta}}+\right)
$$

Processing and forwarding MCP packet: When a node receives a MCP packet, it tests the integrity of the packet. If all the conditions are satisfied, and receiving node is the 
destination, it just simply saves the $\mathrm{MC}$ into cache and sends an $\mathrm{MC}_{A C K}$ packet back to the $\mathrm{N}_{\text {center }}$; otherwise, it updates a reverse route toward the source node and broadcasts the MCP packet to its neighbors. Algorithm 4 shows the procedure for testing and saving the Member Certification at a $\mathrm{N}_{\delta}$ node. When $\mathrm{N}_{\delta}$ receives the MCP packet, it tests the integrity of the packet and whether the MCP is sent by the $\mathrm{N}_{\text {center }}$ node. If all the conditions are satisfied, $\mathrm{N}_{\delta}$ saves the $\mathrm{MC}$ in its cache and unicasts the $\mathrm{MC}_{A C K}$ packet to confirm its certified status to the $N_{\text {center }}$; otherwise, the packet is dropped.

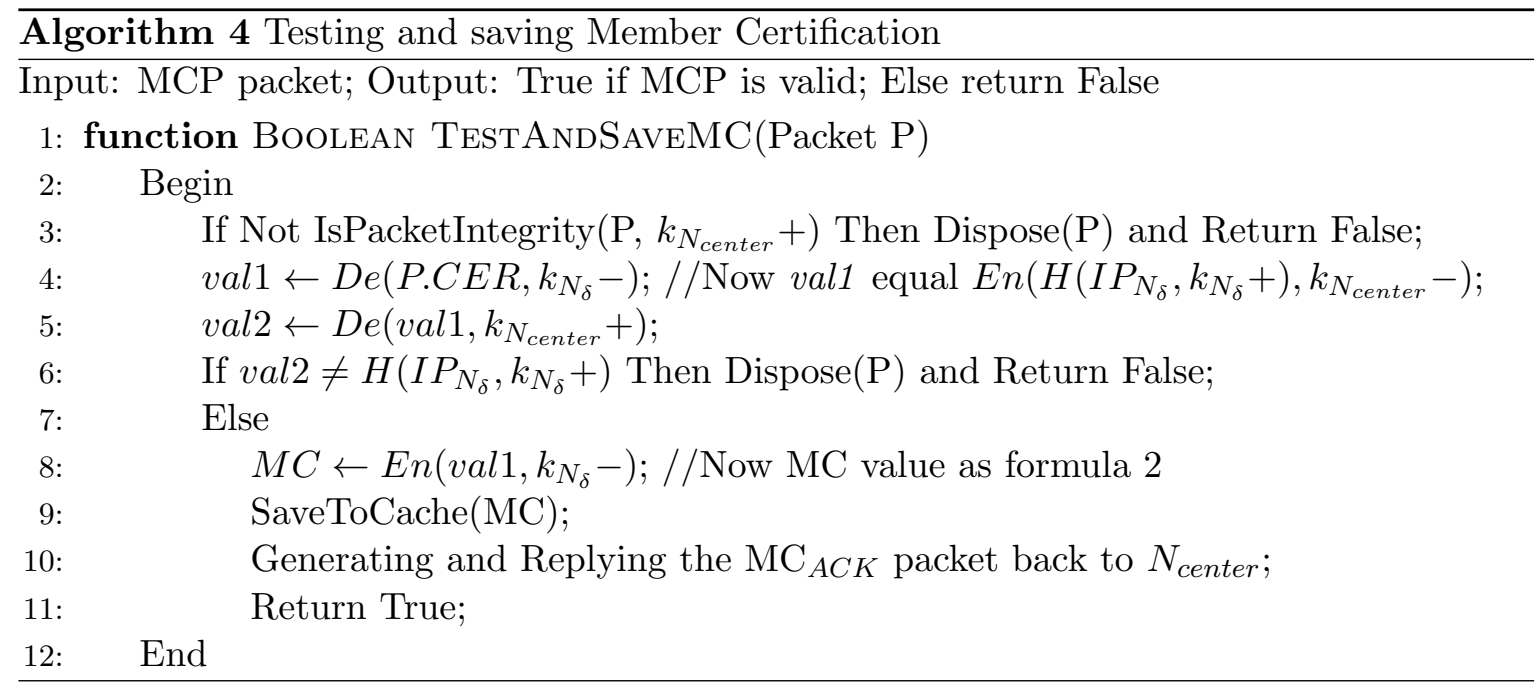

\section{b) Replying the $\mathrm{MC}_{A C K}$ packet}

A certified member node $\mathrm{N}_{\delta}$ is required to send a $\mathrm{MC}_{A C K}$ packet back to confirm its status to the Ncenter. The procedure is similar to the procedure for unicasting the RREP packet of the AODV. The structure of $\mathrm{MC}_{A C K}$ packet is similar as RREP packet and includes three new fields (3NF): ACK, KEY and VC. The ACK field is calculated by (9), the KEY value is the public key of $\mathrm{N}_{\delta}$ and $\mathrm{VC}$ field is the packet integrity.

Generating $M C_{A C K}$ packet: After saving the MC successfully, the node $\mathrm{N}_{\delta}$ unicasts the confirmation packet $\mathrm{MC}_{A C K}$ to back to the $N_{\text {center }}$ as in (8).

$$
N_{\delta} \text { unicasts : } M C_{A C K} \leftarrow\left\{R R E P^{*} \oplus 3 N F\right\}
$$

Where $R R E P^{*}$ is the original RREP packet of AODV routing protocol and extended with the three new fields as described in Figure 6(b).

$$
M C_{A C K} \cdot A C K \leftarrow \operatorname{En}\left(\operatorname{En}\left(H\left(I P_{N_{c e n t e r}}\right), k_{N_{\delta}-}\right), k_{N_{c e n t e r}}+\right)
$$

Processing and forwarding $M C_{A C K}$ packet: When a node receives a $\mathrm{MC}_{A C K}$ packet, it tests the packet integrity. If all the conditions are satisfied and the receiving node is the source, the node just simply updates the successfully provided $\mathrm{MC}$ to the $\mathrm{N}_{\text {center }}$; otherwise, the packet is dropped. Algorithm 5 shows the steps for testing and saving the acknowledgement at the $\mathrm{N}_{\text {center }}$ node. When the $\mathrm{N}_{c e n t e r}$ receives an $\mathrm{MC}_{A C K}$ packet, it tests the integrity of the packet and whether the $\mathrm{MC}_{A C K}$ is sent by the $\mathrm{N}_{\delta}$ node. If all the conditions are 
satisfied, the $\mathrm{N}_{\text {center }}$ updates the confirm status of the node in the PKDB; otherwise, the packet is dropped.

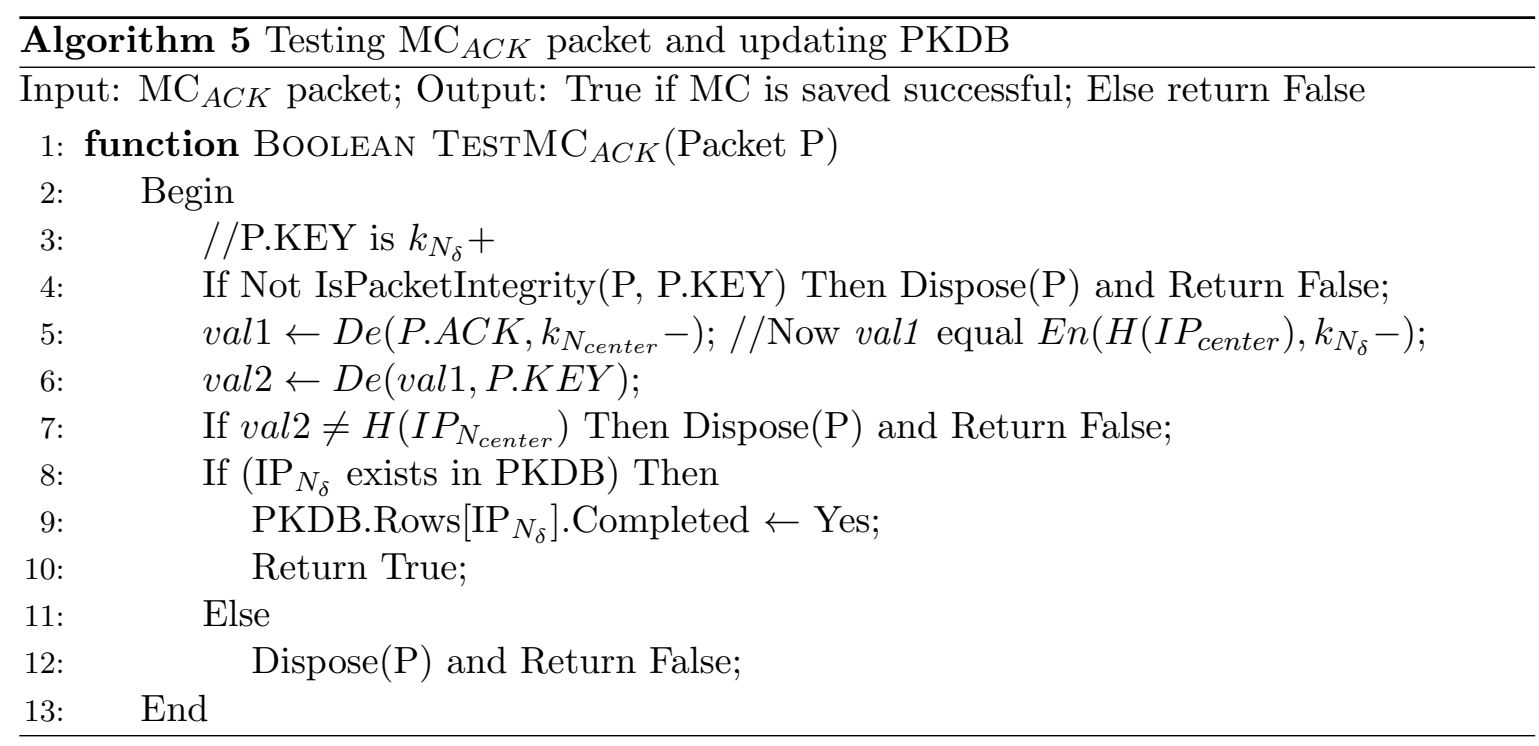

\section{MLAMAN Simulation Results and Performance Analysis}

Using NS2 version 2.35, we simulate MLAMAN and evaluate its performance. The simulation area was a $2000 \mathrm{~m} \mathrm{X} 2000 \mathrm{~m}$ square region, large enough to accommodate an ad hoc network with multiple hops. The simulation time was set at 1000 seconds, long enough for the simulated network to settle down beyond its initial and transitional state.100 normal and 2 malicious nodes were generated for the simulation and 802.11 was used for wireless transmission. A source node sent out constant bit rate (CBR) traffic with packet sizes of 512 bytes at a rate of 2 packets per second. FIFO was used for packet queueing. Table 3 tabulates relevant simulation parameters.

Table 3. Simulation parameters

\begin{tabular}{|l|l|}
\hline Parameters & Setting \\
\hline \hline Simulation area & $2000 \times 2000\left(\mathrm{~m}^{2}\right)$ \\
\hline Simulation times & $1000(\mathrm{~s})$ \\
\hline Number nodes & $102(2$ malicious nodes $)$ \\
\hline Maximum radio range $(\mathrm{R})$ & $250(\mathrm{~m})$ \\
\hline Traffic type & CBR \\
\hline Transport protocol & UDP \\
\hline Data rate & 2 packets per seconds \\
\hline Packet size & 512 bytes \\
\hline Queue type & FIFO (DropTail) \\
\hline Routing protocols & AODV and MLAMAN \\
\hline Hash function $(\mathrm{H})$ & $S H A_{1}$ \\
\hline Prime $(\mathrm{p}, \mathrm{q})$ & 29,31 \\
\hline $\mathrm{T}_{M C}$ & $10($ seconds $)$ \\
\hline
\end{tabular}




\subsection{Wormhole Detection Performance}

We evaluate the wormhole detection performance of the proposed MLAMAN based on tunnel length and mobility speed metrics. Wormhole detection ratio (WDR) is defined as eqn 10 .

$$
W D R=\left(1-\frac{\text { FalsePositive }+ \text { NegativesInstances }}{\text { TotalSevRREQ }+ \text { SecRREP }}\right) * 100 \%
$$

There are 32 scenarios are simulated, each involves 100 normal mobile nodes and 2 malicious nodes. Nodes move in a Random Way Point [26] pattern with a specified maximum speed (MS). Maximum speeds are set at 0,10, 20 and $30 \mathrm{~m} / \mathrm{s}$. 40 pairs of communicating nodes are set up with the source nodes in blue and destination nodes in red. Sources send data at 5 seconds apart from one another, with the first at time zero. Two malicious nodes are positioned near the center of the network with TL (tunnel length) hops between them. TL is set at $1,2,3$, and 4 hops for various simulations. The hop distance is set at $250 \mathrm{~m}$ for the network topology shown in Figure 7.

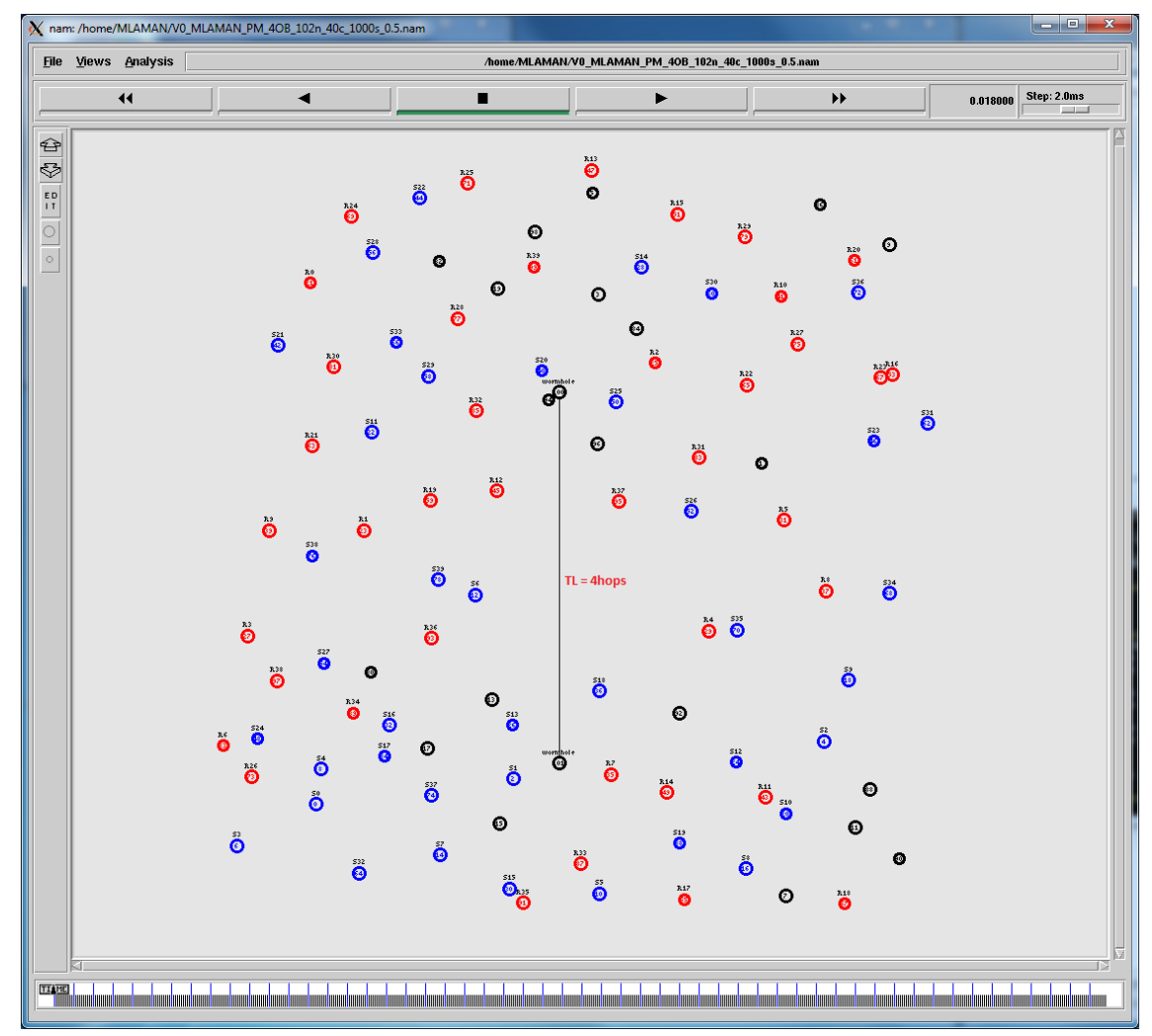

Figure 7. Network topology simulation, 40UDPs connections and Wormhole nodes using 4hops tunnel length

Simulation results in Figure 8 show that MLAMAN has a 100\% the successful detection rate in detecting invalid control route packets for static, stationary network topology where nodes do not move, and over $99.9 \%$ detection rate for all mobile scenarios. Under the PM 
mode during the route discover process, wormhole nodes process control packets just like other normal nodes, MLAMAN detects invalid control route packets (SecRREQs or SecRREPs forwarded by wormhole nodes) by verifying the integrity of these packets using the packet integrity authentication algorithm. Under the HM mode, malicious nodes, on receiving the control packets, simply forwards them to others without processing packet, hence, packet integrity verification and node membership authentication are no longer useful. In this case, MLAMAN detects wormhole node by performing the actual neighbor authentication algorithm at the receiving node to determine if the received SecRREQ (or SecRREP) packet is forwarded by a wormhole node. The weakness of MLA is the actual neighbors authentication using node location to detect malicious node, thus, it can be mistaken in mobility network topology at high speeds.

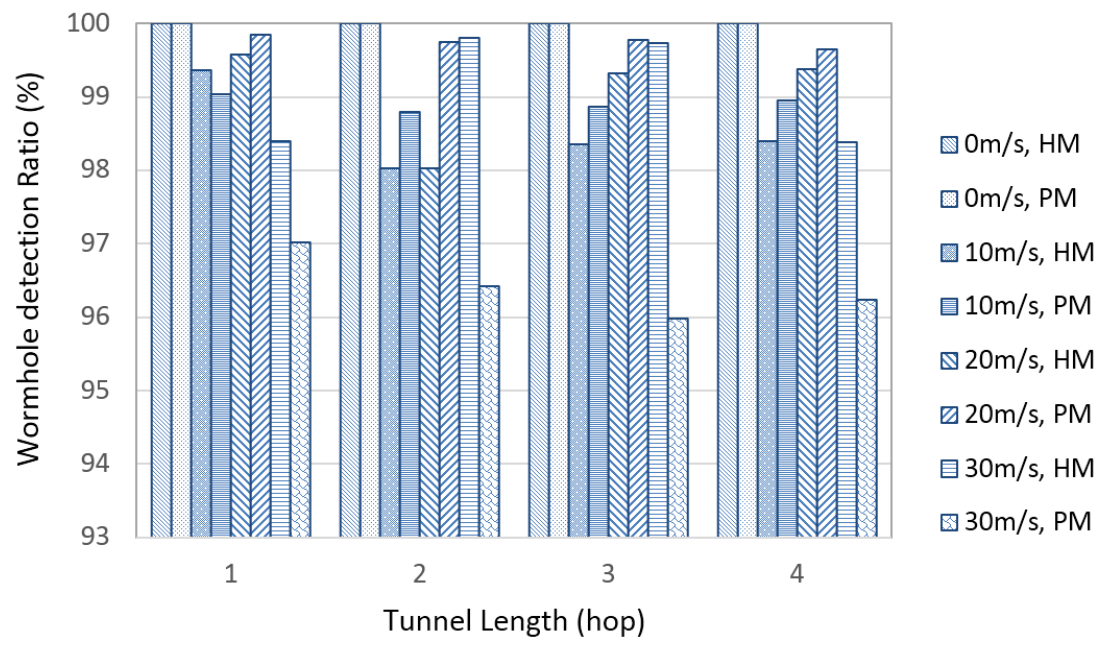

Figure 8. Wormhole detection performance for MLAMAN based on Tunnel Length and Mobility Speed metrics, 40UDPs connections

In this section, we simulate MANETs under different traffic conditions and tunnel lengths and evaluate MLAMAN's performance. Same as before, 32 scenarios are simulated, each has 100 normal mobile nodes and 2 malicious nodes and all of nodes move randomly with a maximum speed of $20 \mathrm{~m} / \mathrm{s}$. Traffic conditions range from light to heavy and are represented by the number of UDP network connections (NCs) between source-destination node pairs from 10 for light traffic to 40 for heavy traffic. Other parameters remain the same as described in Table 3. Simulation results in Figure 9 show that MLA has PM wormhole node detection effectuation is better than HM wormhole node. The successful detection ratio over $98.03 \%$ of wormhole nodes using both of HM and PM modes for all simulation scenarios. With TTHCA, the detection performance is excellent for long tunnels, but it degrades to less than $90 \%$ [12] when the tunnel length is less than 5 due to the difficulty in measuring short the packet transversal times. This fact also implies that TTHCA may not perform well when nodes are moving at various speeds. The detection results in simulation results in Figure 8 and Figure 9 confirm that MLA outperformed TTHCA and other methods [9][12] for short tunnel length and high mobility speed simulation scenarios. 


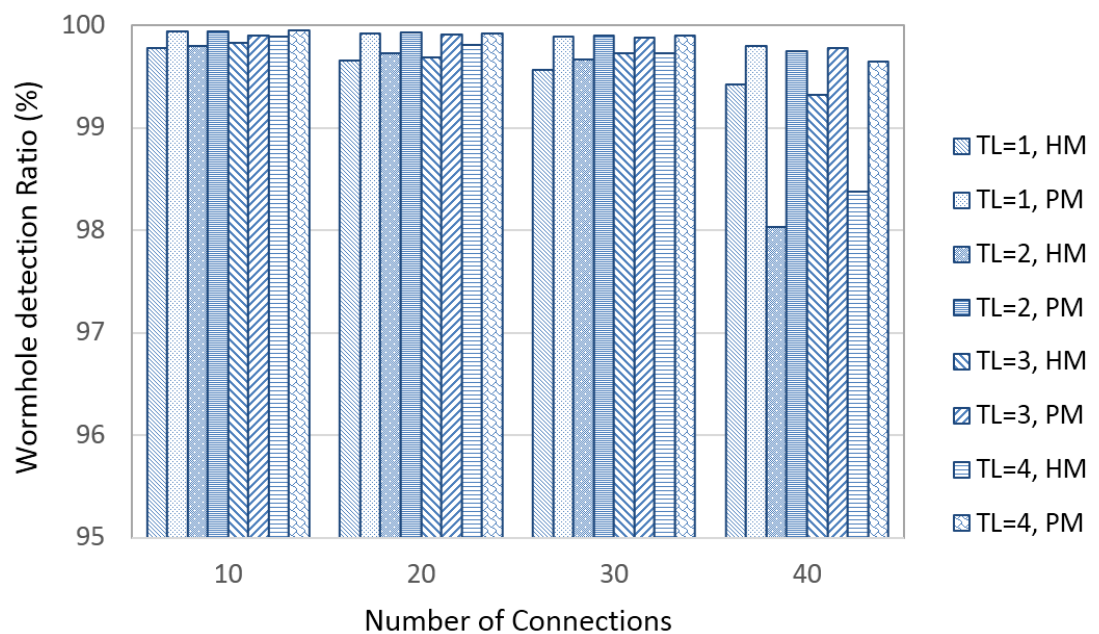

Figure 9. Wormhole detection performance for MLAMAN based on Number UDP connection and Tunnel Length metric, $20 \mathrm{~m} / \mathrm{s}$ mobility speeds maximum

\subsection{Packet overhead and Packet delivery ratio}

In this section we analyze the packet overhead (PO) for providing the node membership certification and the packet delivery ratio (PDR) in a normal network topology as shown in eqn 11 and 12 .

$$
\begin{gathered}
P O=\text { Total of } M C P+M C_{A C K} \\
P D R=\left(\frac{\text { Number of packets delivered successfully }}{\text { Total of packtes are sent from source }}\right) * 100 \%
\end{gathered}
$$

Four specific scenarios are simulated, has all of them assume 20 pairs of communicating nodes with the first source node sending data at time zero and the rest sending data at 5 -second intervals.

The first scenario simulates AODV protocol for 100-node MANET. The second scenario simulates MLAMAN for 100-node MANET and used with 100 member nodes in the PKDB database;

The third scenario simulates MLAMAN for100-node MANET with 80 member nodes from 0 to 79 identified in PKDB;

The fourth scenario simulates MLAMAN for 100-node MANET with 80 member nodes from 0 to 79 identified in PKDB and 20 new member nodes are installed into PKDB at 200th seconds.

Figure 10 shows that MLAMAN requires 80 seconds and an overhead of 19,645 of packets (CMP and MCACK) to provide MC for all 100 member nodes listed in the PKBD database. The overheads are 70 seconds and 18,056 packets for 80 member nodes. For the fourth scenario, total packet overhead of MLAMAN is 20,412 packets and 230 seconds for completing the member certification process. 


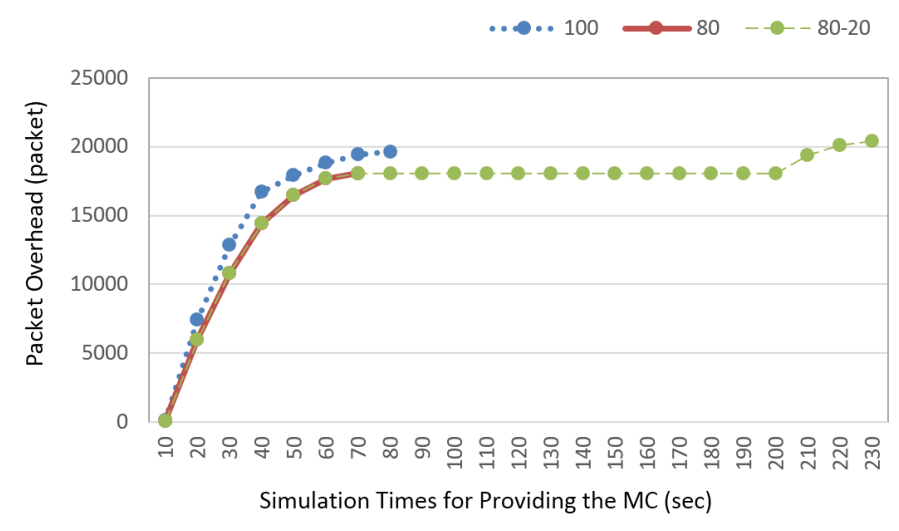

Figure 10. Packet overhead of MLAMAN protocol to provide MC for member nodes

Figure 11 shows that the packet delivery ratio of AODV is better than MLAMAN with all member nodes listed in the PKBD. With MLAMAN, most of the nodes cannot participate in the route discover process at the early stage of the operation as they have not yet been certified and hence packets cannot be delivered to their destination. As time progresses, more and more nodes are certified resulting in higher number of successful packet delivery. The MC process completes after 80 seconds providing all listed member nodes are operational. At the end of the simulation, the PDR for MLAMAN is $59.13 \%$ compared to $72.94 \%$ for the AODV for this scenario. With the scenario where 80 member nodes are installed in PKBD, PDR of MLAMAN is reduced to $23.89 \%$ due to the fact that around $50 \%$ of the destination nodes was not in PKDB. Final scenario, PDR for MLAMAN is improved to $52.1 \%$ eventually as 20 new member nodes are added into PKDB at time 200s.

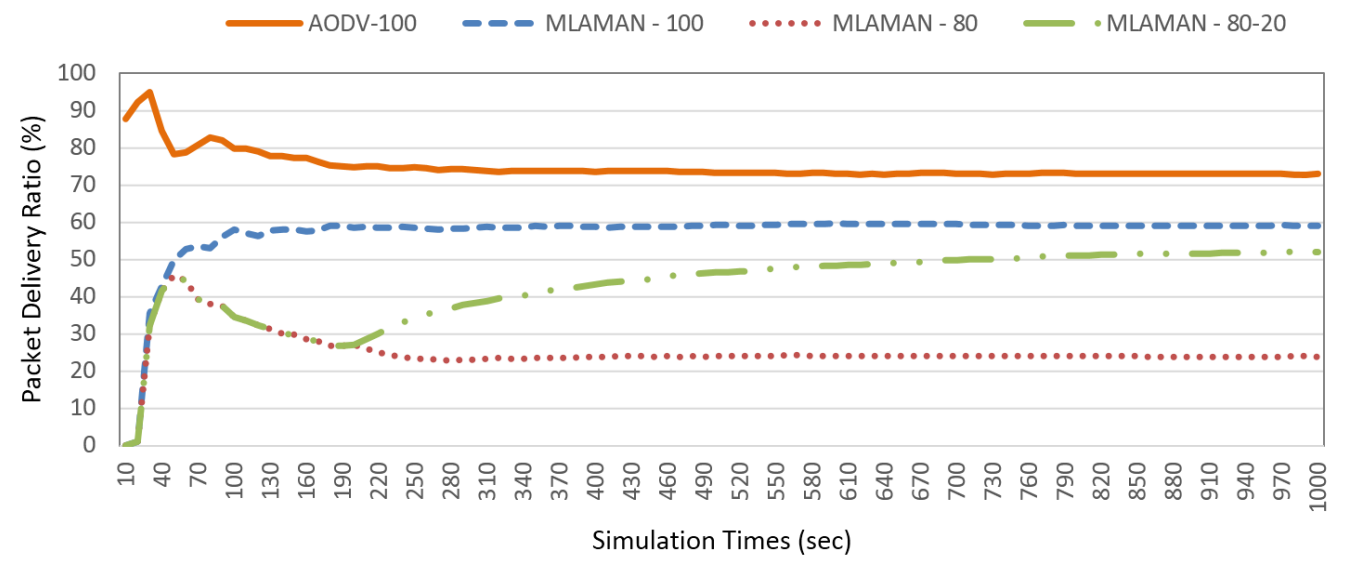

Figure 11. Comparison of packet delivery ratio of AODV and MLAMAN protocols in normal network topology 


\subsection{Route Discovery Time}

To evaluate the route discovery time of MLAMAN compared to the original AODV protocol. MLAMAN requires every mobile nodes, on a hop-by-hop basis, to test the integrity of the control packets SecRREQ and SecRREP, verify the membership of the sending nodes as well as their neighborhood status for wormhole detection, it is expected that the route discovery time of MLAMAN be higher than that of AODV. The simulation results in Figure 12 are based on the average route discovery delay for scenario using 100 normal mobile nodes. It is clear that when the network traffic increases the route discovery time increases.

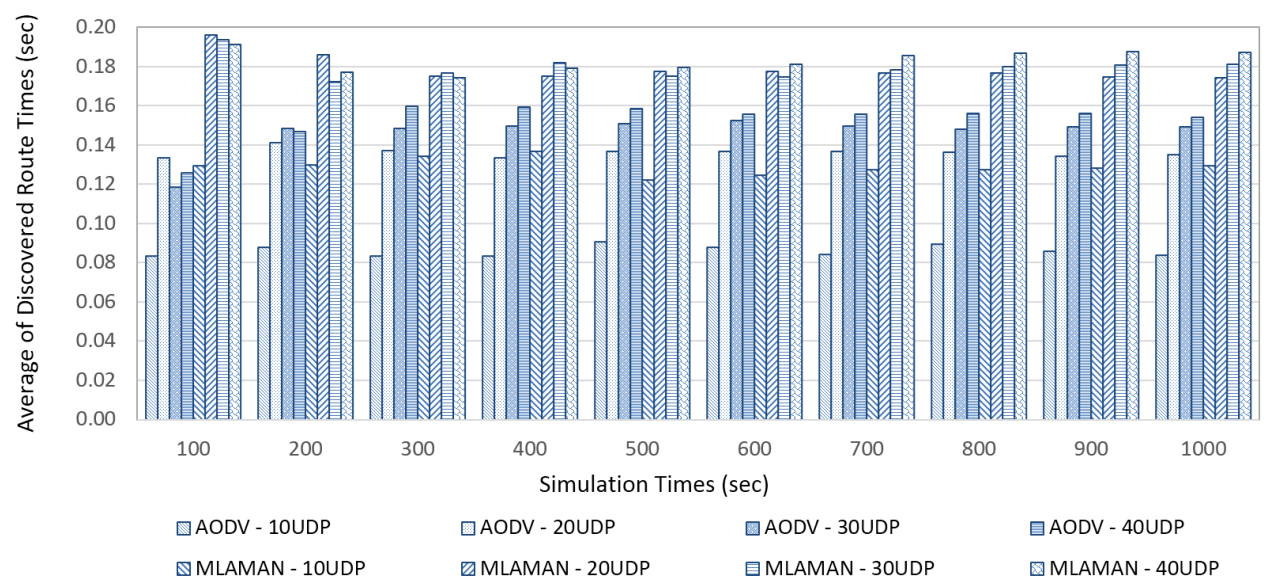

Figure 12. Route discovery delay of AODV and MLAMAN protocols in normal network topology

\section{Conclusions and Future Works}

We proposed MLAMAN, a novel model that deployed multi-level authentication and routing protocol to prevent wormhole attacks in MANETs. MLAMAN verifies the integrity and the authenticity of a routing control packet on a hop-by-hop basis. It also utilizes positioning information of the sending and receiving nodes to judge their neighborhood relationship. Simulation results demonstrated that the MLAMAN was very robust against wormhole attacks. For a static network topology, it was 100\% successful in detecting wormhole attacks. For a dynamic and mobile topology where a minimum tunnel length of 1 hop and with a maximum node moving speed of $30 \mathrm{~m} / \mathrm{s}$ it achieves a successful wormhole detection rate of over $95.98 \%$ in both Hidden and Participation Modes. To support MLAMAN, a management protocol was introduced to provide node membership certification to prevent malicious nodes from joining the network with the fake keys.

Our next step is to setup MLAMAN with large key to improve the security performance using TLS library [17] and evaluate the security capability of MLAMAN through comprehensive simulations, taking into account practical scenarios including network sizes and node's speeds. 


\section{REFERENCES}

[1] H. Chiu and K. Wong Lui, "DelPHI: Wormhole detection mechanism for Ad hoc Wireless Networks," in International Symposium on Wireless Pervasive Computing Proceedings, 2006, pp. 6 -11 .

[2] W. Diffie, W. Diffie, and M. E. Hellman, "IEEE Transactions on Information Theory," vol. 22, no. 6,1976 , pp. $644-654$.

[3] A. Eiman and M. Biswanath, "A survey on routing algorithms for Wireless Ad-Hoc and Mesh Networks," Computer Networks, vol. 56, no. 2, pp. 940 - 965, 2012.

[4] S. Gurung and S. Chauhan, "A novel approach for mitigating gray hole attack in MANET," Wireless Networks, pp. 1 - 15, 2016.

[5] J. Hoebeke, I. Moerman, B. Dhoedt, and P. Demeester, "An overview of Mobile Ad hoc Networks: applications and challenges," Journal of the Communications Network, vol. 3, no. 3, pp. 60 - 66, 2004.

[6] Y. C. Hu, A. Perrig, and D. B. Johnson, "Packet leashes: A defense against wormhole attacks in Wireless Networks," in IEEE INFOCOM 2003. Twenty-second Annual Joint Conference of the IEEE Computer and Communications Societies (IEEE Cat. No.03CH37428), vol. 3, 2003, pp. $1976-1986$.

[7] T. Issariyakul and E. Hossain, Introduction to Network Simulator NS2. Springer, 2009.

[8] V. M. Jan, W. Ian, and K. S. Winston, "Security threats and solutions in MANETs: A case study using AODV and SAODV," Journal of Network and Computer Applications, vol. 35, no. 4, pp. $1249-1259,2012$.

[9] S. M. Jen, C. S. Laih, and W. C. Kuo, "A Hop-Count Analysis Scheme for Avoiding Wormhole Attacks in MANET," Sensors, vol. 9, no. 6, pp. 5022 - 5039, 2009.

[10] D. B. Johnson and D. A. Maltz, Dynamic Source Routing in Ad Hoc Wireless Networks. Boston, MA: Springer US, 1996, pp. 153-181.

[11] P. Jones. US secure hash algorithm 1 (SHA1). [Online]. Available: https://tools.ietf.org/html/ rfc3174

[12] J. Karlsson, L. S. Dooley, and G. Pulkkis, "A New MANET Wormhole Detection Algorithm Based on Traversal Time and Hop Count Analysis," Sensors, vol. 11, no. 12, pp. 11122 - 11 140, 2011.

[13] — - "Identifying Time Measurement Tampering in the Traversal Time and Hop Count Analysis (TTHCA) Wormhole Detection Algorithm," Sensors, vol. 13, no. 5, pp. 6651-6668, 2013.

[14] S. Khurana and N. Gupta, "End-to-end protocol to secure ad hoc networks against wormhole attacks," Security and Communication Networks, vol. 4, no. 9, pp. $994-1002,2011$.

[15] L. Lazos, R. Poovendran, C. Meadows, P. Syverson, and L. W. Chang, "Preventing wormhole attacks on Wireless Ad hoc Networks: A graph theoretic approach," in IEEE Wireless Communications and Networking Conference, 2005, vol. 2, 2005, pp. 1193 - 1199.

[16] L. Lazos and R. Poovendran, "SeRLoc: Secure Range-independent Localization for Wireless Sensor Networks," in Proceedings of the 3rd ACM Workshop on Wireless Security, 2004, pp. 21 -30 . 
[17] T. library. RSA source code. [Online]. Available: https://tls.mbed.org/rsa-source-code

[18] G. Z. Manel, "Secure Ad Hoc On-demand Distance Vector Routing," ACM SIGMOBILE Mobile Computing and Communications Review, vol. 6, no. 3, pp. 106 - 107, 2002.

[19] E. C. Ngai, J. Liu, and M. R. Lyu", "An efficient intruder detection algorithm against sinkhole attacks in wireless sensor networks," Computer Communications, vol. 30, no. 11, pp. 2353 $2364,2007$.

[20] L. T. Ngoc and V. T. Tu, "Whirlwind: A new method to attack Routing Protocol in Mobile Ad hoc Network," International Journal of Network Security, vol. 19, no. 5, pp. 832 - 838, 2017.

[21] C. E. Perkins and E. M. Royer, "Ad-hoc On-Demand Distance Vector Routing," in Proceedings of the Second IEEE Workshop on Mobile Computer Systems and Applications, 1999, pp. 90 100 .

[22] R. D. Pietro, S. Guarino, N. Verde, and J. Domingo-Ferrer, "Security in Wireless Ad-hoc Networks - A survey," Computer Communications, vol. 51, pp. 1 - 20, 2014.

[23] K. Sanzgiri, B. Dahill, B. N. Levine, C. Shields, and E. M. Belding-Royer, "A secure routing protocol for Ad hoc Networks," in 10th IEEE International Conference on Network Protocols, 2002. Proceedings, 2002, pp. $78-87$.

[24] R. Thillaikarasi and S. M. S. Bhanu, "An Efficient DSR Protocol to Detect Blackhole Attacks in WMN Using Cross Layer Approach," Wireless Personal Communications, 2017.

[25] V. T. Tu and L. T. Ngoc, "SMA 2 AODV: Routing Protocol Reduces the Harm of Flooding Attacks in Mobile Ad Hoc Network," Journal of Communications, vol. 12, no. 7, pp. 371 - 378, 2017.

[26] J. Yoon, M. Liu, and B. Noble, "Random waypoint considered harmful," in IEEE INFOCOM 2003. Twenty-second Annual Joint Conference of the IEEE Computer and Communications Societies (IEEE Cat. No.03CH37428), vol. 2, 2003, pp. 1312 - 1321.

\section{Authors information}

Tu T. Vo is an associate professor in the Faculty of Information Technology, Hue University of Sciences, Hue University. He received B.E. degree in Physics from Hue University in 1987 and $\mathrm{PhD}$ degree in computer science from Institute of Information Technology, Vietnam Academy of Science and Technology in 2005. His fields of interesting are network routing, analysis and evaluation of network performance, security wireless Ad hoc Network.

Ngoc T. Luong is working in the Faculty of Mathematics and Informatics Teacher Education, Dong Thap University. He received B.E. degree in Computer Science from Dong Thap University in 2007 and M.A. degree in Computer Science from Hue University of Sciences in 2014. He is a PhD student in Hue University of Sciences now. His fields of interesting are analysis and evaluation of network performance, security wireless Mobile Ad hoc Network.

Doan Hoang is a Professor in the School of Computing and Communications, University of Technology Sydney (UTS). Doan's research focuses on cyber security and quality of service of software-defined infrastructures (Software-Define Networks (SDN)), Cloud, and Internet of Things (IoT). In particular, his current research projects include: Resource optimization 
in SDN environment, Software-defined architecture for provisioning IoT applications on demand, Network Functions Virtualization (NFV), SDN and virtualization for Cyber Security, Security metrics and maturity models for Cloud, and Trust assessment model for Personal Space IOTs. He is also into establishing infrastructure for real-time accessing, distribution, and protection of big assistive healthcare data. 